\title{
25-Gb/s Transmission Over 2.5-km SSMF by Silicon MRR Enhanced 1.55-mu m III-V/SOI DML
}

Cristofori, Valentina; Da Ros, Francesco; Ozolins, Oskars; Chaibi, Mohamed E.; Bramerie, Laurent; Ding, Yunhong; Pang, Xiaodan; Shen, Alexandre; Gallet, Antonin; Duan, Guang-Hua

Total number of authors:

16

Published in:

I E E E Photonics Technology Letters

Link to article, DOI:

10.1109/LPT.2017.2700497

Publication date:

2017

Document Version

Early version, also known as pre-print

Link back to DTU Orbit

Citation (APA):

Cristofori, V., Da Ros, F., Ozolins, O., Chaibi, M. E., Bramerie, L., Ding, Y., Pang, X., Shen, A., Gallet, A., Duan, G-H., Hassan, K., Olivier, S., Popov, S., Jacobsen, G., Oxenløwe, L. K., \& Peucheret, C. (2017). 25-Gb/s Transmission Over 2.5-km SSMF by Silicon MRR Enhanced 1.55-mu m III-V/SOI DML. I E E E Photonics Technology Letters, 29(12), 960-963. https://doi.org/10.1109/LPT.2017.2700497

\section{General rights}

Copyright and moral rights for the publications made accessible in the public portal are retained by the authors and/or other copyright owners and it is a condition of accessing publications that users recognise and abide by the legal requirements associated with these rights.

- Users may download and print one copy of any publication from the public portal for the purpose of private study or research.

- You may not further distribute the material or use it for any profit-making activity or commercial gain

- You may freely distribute the URL identifying the publication in the public portal 


\title{
25-Gb/s Transmission Over 2.5-km SSMF by Silicon MRR Enhanced 1.55- $\mu \mathrm{m}$ III-V/SOI DML
}

\author{
Valentina Cristofori, Francesco Da Ros, Oskars Ozolins, Mohamed E. Chaibi, Laurent Bramerie, Yunhong Ding, \\ Xiaodan Pang, Alexandre Shen, Antonin Gallet, Guang-Hua Duan, Karim Hassan, Ségolene Olivier, \\ Sergei Popov, Gunnar Jacobsen, Leif K. Oxenløwe, Christophe Peucheret
}

\begin{abstract}
The use of a micro-ring resonator (MRR) to enhance the modulation extinction ratio and dispersion tolerance of a directly modulated laser (DML) is experimentally investigated with a bit rate of $25 \mathrm{~Gb} / \mathrm{s}$ as proposed for the next generation data center communications. The investigated system combines a $11-\mathbf{G H z} 1.55-\mu \mathrm{m}$ directly modulated hybrid III-V/SOI DFB laser realized by bonding III-V materials (InGaAlAs) on a siliconon-insulator (SOI) wafer and a silicon MRR also fabricated on SOI. Such a transmitter enables error-free transmission $(B E R<$ $10^{-9}$ ) at $25 \mathbf{G b} / \mathbf{s}$ data rate over $2.5-\mathrm{km}$ SSMF without dispersion compensation nor forward error correction (FEC). As both laser and MRR are fabricated on the SOI platform, they could be combined into a single device with enhanced performance, thus providing a cost-effective transmitter for short reach applications.
\end{abstract}

Index Terms-Photonic integrated circuits, Resonators filters, Optical transmitters.

\section{INTRODUCTION}

$\mathbf{T}$ HE traffic in data centers has been steadily growing to fulfill the ever increasing customer demand and this has pushed research towards finding energy- and cost-effective solutions capable of reaching modulation speeds higher than the current standard of $10 \mathrm{~Gb} / \mathrm{s}$. Transmission at $25 \mathrm{~Gb} / \mathrm{s}$ over standard single mode fiber (SSMF) has been proposed as next target to be included in the IEEE 802.3 standard [1]. Furthermore, physical space in data centers being a scarce resource, the focus has been directed towards finding integrated and compact solutions that could still satisfy the energy and cost requirements. In this perspective, directly modulated lasers (DMLs) have been recognized as good candidates to address these needs [2].

DMLs are, in fact, considered as promising alternatives to external modulation for short reach applications due to their lower cost and power consumption. High-speed DMLs have already been demonstrated, showing their capability to operate

V. Cristofori, F. Da Ros, Y. Ding and Leif K. Oxenløwe are with the Department of Photonics Engineering, Technical University of Denmark, Kongens Lyngby, 2800 Denmark, e-mail: vari@fotonik.dtu.dk.

O. Ozolins, X. Pang, G. Jacobsen are with NETLAB, Acreo Swedish ICT, Kista, 16425, Sweden

M. E. Chaibi, L. Bramerie and C. Peucheret are with FOTON Laboratory, CNRS UMR 6082, ENSSAT, University of Rennes 1, Lannion, 22305 France.

A. Shen, A. Gallet and G.-H. Duan are with III-V Lab, a joint lab of Nokia, Thales and CEA, 1 Avenue A. Fresnel, 91767 Palaiseau, France.

K. Hassan and S. Olivier are with CEA-Leti, 17 rue des Martyrs, 38054 Grenoble, France.

S. Popov is with School of ICT, KTH Royal Institute of Technology, Electrum 229, Kista, 16440, Sweden

Manuscript received February 13, 2017; revised , 2017. beyond $10 \mathrm{~Gb} / \mathrm{s} \mathrm{[3],} \mathrm{[4].} \mathrm{However,} \mathrm{the} \mathrm{major} \mathrm{challenges} \mathrm{to} \mathrm{the}$ deployment of DMLs are the low modulation extinction ratio (ER) and the frequency chirp induced by the direct modulation of the laser current, which decreases the dispersion tolerance. A potential solution to the latter consists in operating in the O-band (1300 nm). However, the higher fiber loss decreases the power budget of the system. Operation in the C-band (1550 nm), instead, would be highly desirable.

To overcome these challenges and thus allow operation in the C-band, several techniques have been proposed and successfully demonstrated, such as the use of passive filtering for chirp management [5] or simply ER and dispersion tolerance enhancement by either a delay interferometer [6] or by a micro-ring resonator (MRR) [7]-[10]. An advantage of using MRRs as notch filters for ER enhancement is that they can be fabricated on the silicon on insulator (SOI) platform in a compact way. Furthermore, it has already been demonstrated that, by using the drop port of the MRR, the laser emission wavelength and the MRR resonance can be locked in an effective way [11]. Additionally, thanks to the progress in integration of III-V materials on the SOI platform [12], it has been recently shown that hybrid DFB lasers can be integrated on the same chip with a silicon MRR, resulting in a significant improvement of the DMLs performance [10]. The combination of hybrid III-V lasers on SOI and MRR is, therefore, a promising technique for the cost-effective implementation of compact transmitters for short reach applications.

In this letter, we report on an all-on-silicon transmitter operating at the target bit rate of $25 \mathrm{~Gb} / \mathrm{s}$. This is achieved by combining a directly modulating III-V/Si hybrid DFB laser and an optimized silicon MRR filter. Error-free $\left(\mathrm{BER}<10^{-9}\right)$ direct detection of on-off keying (OOK) signals after transmission over $2.5 \mathrm{~km}$ of SSMF is demonstrated without need for dispersion compensation nor forward error correction (FEC).

\section{DESIGN AND FABRICATION}

\section{A. III-V SOI DFB Laser}

The structure of the hybrid III-V/SOI DFB is shown in Fig. 11. The fabrication makes use of a 200-mm CMOS line, starting with SOI wafers having a silicon top layer thickness of typically $440 \mathrm{~nm}$. A phase-shifted Bragg grating is etched on the top silicon waveguide layer, then a passive rib waveguide is formed by etching the top silicon layer. The waveguide is optimized for coupling with a III-V waveguide that will be aligned on top of the silicon waveguide in a later step. After 


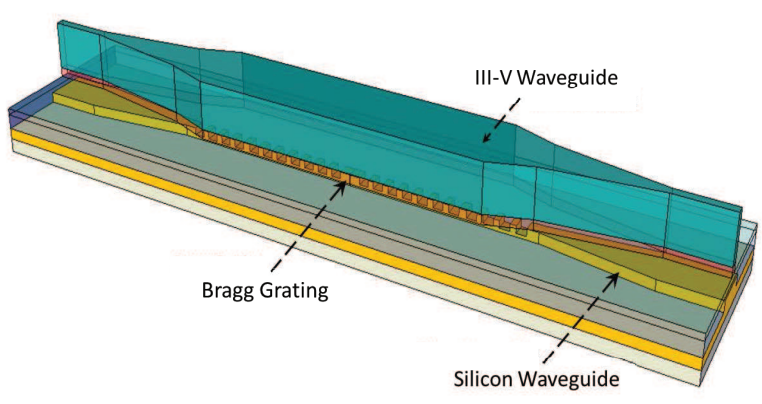

Fig. 1. Hybrid III-V/SOI DFB laser structure.

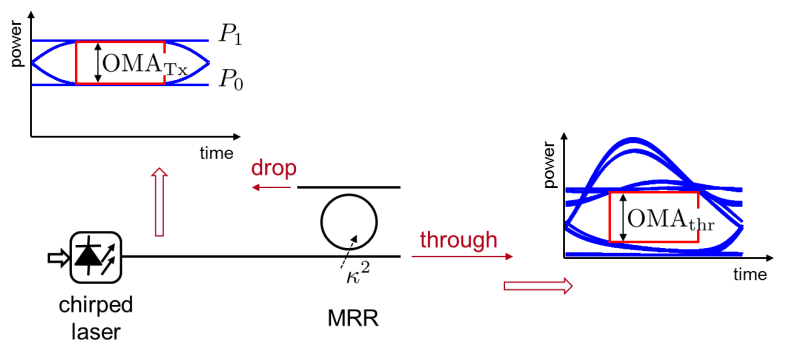

Fig. 2. MRR optimization procedure based on comparison of the OMA at the DML output $\left(\mathrm{OMA}_{\mathrm{Tx}}\right)$ to the OMA after filtering at the through port of the MRR $\left(\mathrm{OMA}_{\mathrm{thr}}\right)$ for different values of peak-to-peak adiabatic chirp and MRR power coupling coefficient $\kappa^{2}$.

this etching step, the remaining silicon layer has a thickness of $220 \mathrm{~nm}$. For the passive circuitry, additional etching steps are applied to form the strip waveguides and other elements such as the vertical output coupler. A silica layer is deposited and a chemical-mechanical polishing is applied in order to planarize the surface of the SOI wafer. In parallel, a $2^{\prime \prime}$ InP wafer containing multiple quantum well layers is grown and bonded onto the SOI wafer. After wafer bonding and InP substrate removal, a combination of wet and dry etching is used to etch through the InGaAlAs contact layer and the InP p-doped waveguide cladding layer. The active waveguide is then encapsulated with benzocyclobutene (DVS-BCB). A $\mathrm{Ti} / \mathrm{Pt} / \mathrm{Au}$ alloy is used for metallization of both $\mathrm{p}$ - and $\mathrm{n}$ - type contacts. Finally, the fabricated laser is ready for wafer level probe testing using the vertical grating coupler as access for the optical probe.

\section{B. Silicon Micro-ring Resonator}

The MRR parameters were optimized numerically, as illustrated in Fig. 2. The optimization target was to maximize the optical modulation amplitude (OMA) of a 25-Gb/s signal affected by transient and adiabatic chirp after filtering it with the in-to-through transfer function of a MRR having a freespectral range (FSR) of $100 \mathrm{GHz}$. The FSR value was chosen to potentially allow for simultaneous filtering of multiple FSRspaced WDM channel in a $N \times 25 \mathrm{~Gb} / \mathrm{s}$ laser array. For a given silicon waveguide structure (hence group index $n_{g}$ ) and fabrication technology (hence loss value, taken here equal to $1 \mathrm{~dB} / \mathrm{cm}$ ), the only free parameter is the power coupling coefficient $\kappa^{2}$ between the ring and the straight waveguides, taken equal at the through and drop ports. An ideal non-returnto-zero (NRZ) OOK signal with extinction ratio of $2 \mathrm{~dB}$ was

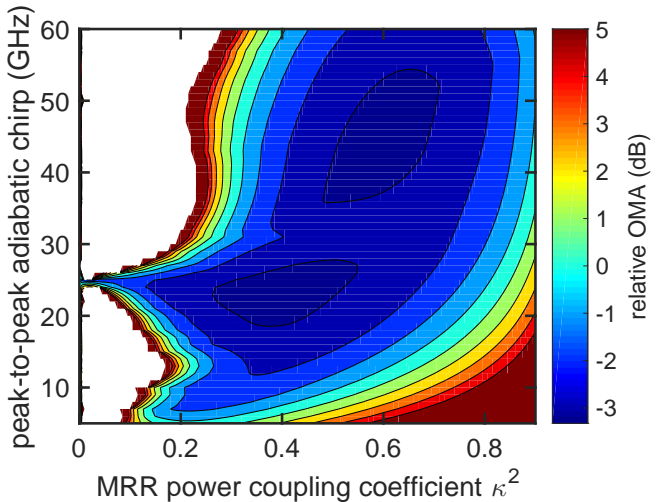

Fig. 3. Relative OMA as a function of MRR power coupling coefficient $\kappa^{2}$ and peak-to-peak adiabatic chirp of the DML: negative values show the improvement provided by the MRR. The calculations have been performed here with $\alpha=2$, but since the peak-to-peak adiabatic chirp depends on the product $\alpha \kappa_{c}$, the actual value of the linewidth enhancement factor does not significantly impact the results.

generated, and its frequency chirp was modeled according to [13]

$$
\Delta \nu=\frac{\alpha}{4 \pi}\left[\frac{\mathrm{d} P(t)}{\mathrm{d} t}+\kappa_{c} P(t)\right],
$$

where $\alpha$ is the linewidth enhancement factor of the laser, $\kappa_{c}$ its adiabatic chirp coefficient, and $P(t)$ is the emitted power. A 2-dB extinction ratio is a typical value for a DML operated at high bias currents in order to benefit from the enhanced bandwidth of the laser and reduced impact of relaxation oscillations, which are damped for high laser driving currents.

Some typical results of the optimization process are represented in Fig. 3, where the relative OMA, defined as the ratio of the OMA at the DML output to the OMA after filtering, i.e. $10 \log \left(\mathrm{OMA}_{\mathrm{Tx}} / \mathrm{OMA}_{\mathrm{thr}}\right)$ according to the notations of Fig. 2 , was calculated as a function of MRR power coupling coefficient $\kappa^{2}$ and peak-to-peak adiabatic chirp $\alpha \kappa_{c} \mathrm{OMA}_{\mathrm{Tx}} / 4 \pi$. In order to account for signal distortion due to filtering, the OMA is evaluated in a conservative way by considering the height of a rectangle of width $1 / 2 R_{b}$, where $R_{b}$ is the bit rate, that fits within the filtered eye diagram. It can be seen in Fig. 3 that, for a given adiabatic chirp value, the OMA can be improved over a wide range of MRR power coupling coefficient values. The peak-to-peak adiabatic chirp measured when modulating the hybrid DFB laser at $25 \mathrm{~Gb} / \mathrm{s}$ is approximately $13 \mathrm{GHz}$, resulting in an optimum value of $\kappa^{2}$ of approximately 0.4 . This value is therefore the target for the power coupling coefficient when designing the MRR. These optimal MRR parameters are translated into physical dimensions for the fabrication of the MRR by modeling it through the coupled mode theory [14]. The desired FSR of $100 \mathrm{GHz}$ is obtained by choosing a ring diameter of $120 \mu \mathrm{m}$.

The MRR fabrication started from an SOI wafer with a top silicon thickness of $250 \mathrm{~nm}$ over a $3-\mu \mathrm{m}$ buried silicon dioxide layer. Electron-beam (EB) lithography and inductively coupled plasma reactive ion etching were used to define the micro-ring structure shown in Fig. 4 (a). Note that the use of EB lithography is not strictly necessary for the dimensions of the device; deep-UV lithography could also be employed facilitating the fabrication of the device in a standard CMOS process. Plasma- 
(a)

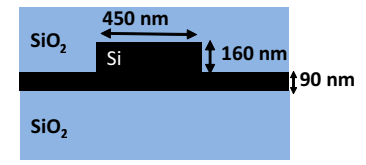

(b)

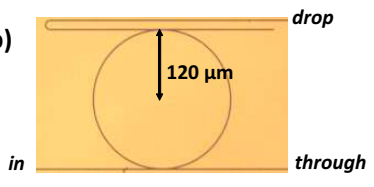

Fig. 4. (a) MRR cross-section and (b) MRR microscope picture.
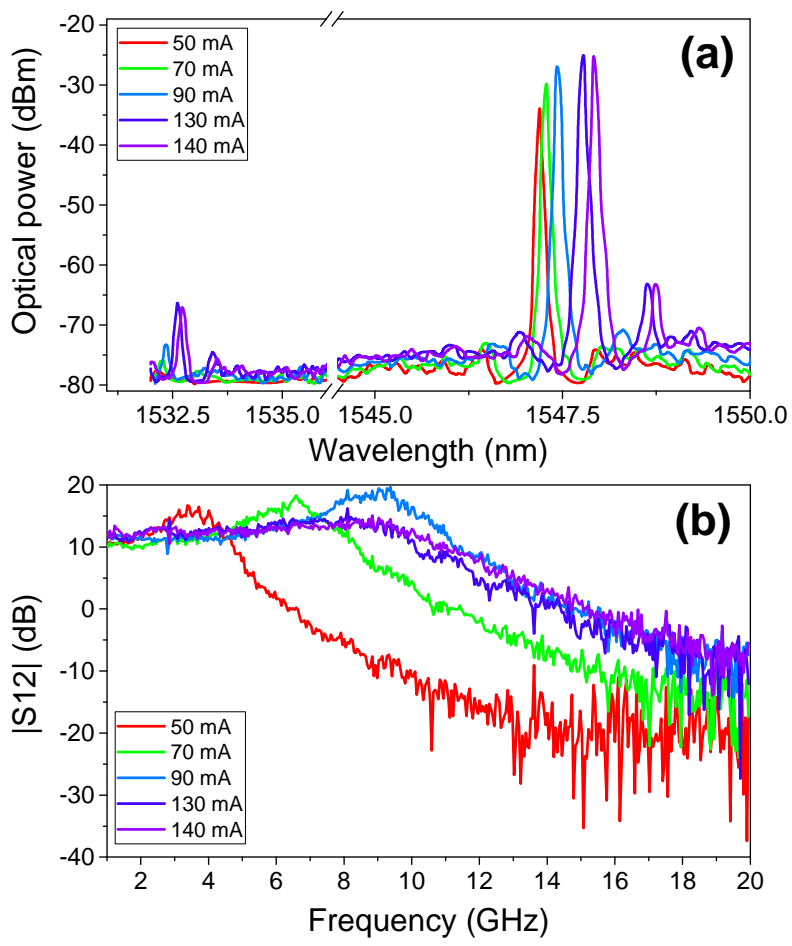

Fig. 5. (a) Optical spectra of the hybrid DFB laser for different bias currents and (b) the corresponding small-signal amplitude modulation responses.

enhanced chemical vapor deposition (PECVD) was then used to deposit a silica cladding top layer. The rib waveguide has a depth of $160 \mathrm{~nm}$ and its width is $450 \mathrm{~nm}$. The gap width between the straight waveguide and the ring waveguide is $300 \mathrm{~nm}$, corresponding to an estimated power coupling coefficient $\kappa^{2}=0.45$. A microscope picture of the device is shown in Fig. 4 (b). Apodized grating couplers [15] are implemented at the in, through and drop ports to couple light in and out of the MRR and the in-to-through total insertion loss of the MRR away from resonance is $9 \mathrm{~dB}$. A significant part of the loss could be avoided by integrating the MRR with the laser. The main loss contribution is indeed due to the loss in the grating couplers estimated to be approximately $4 \mathrm{~dB}$ per coupler. The measured MRR Q-factor is $3.8 \times 10^{4}$.

\section{Hybrid DFB Laser Static Characterization}

First a static characterization of the laser is performed and the optical spectra of the hybrid DFB and its small-signal frequency responses for different bias currents between $50 \mathrm{~mA}$ and $140 \mathrm{~mA}$ are measured and shown in Fig. 5 (a) and (b), respectively. From the spectra in Fig. 5] (a), a side mode suppression ratio above $40 \mathrm{~dB}$ is estimated, showing good single mode performance. The $3-\mathrm{dB}$ modulation bandwidth is extracted from the $S_{21}$ curves in Fig. 5(b) and measured to be approximately $11 \mathrm{GHz}$ for bias currents between $130 \mathrm{~mA}$

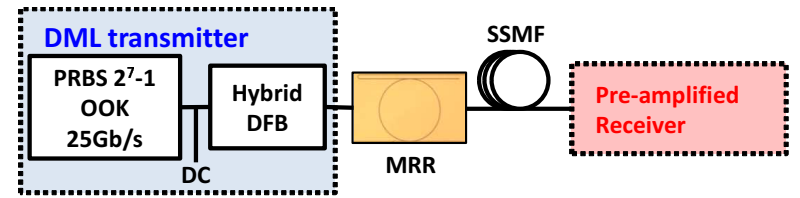

Fig. 6. Experimental setup for dynamic characterization of the DFB laser at $25 \mathrm{~Gb} / \mathrm{s}$.

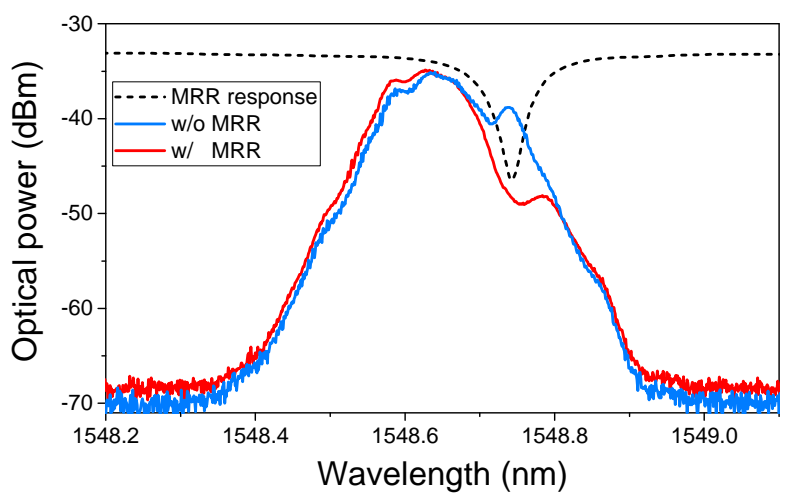

Fig. 7. Optical spectra of the DFB laser modulated at $25 \mathrm{~Gb} / \mathrm{s}$ with and without the MRR filtering. The measured MRR transfer function is also represented by the dashed line.

and $140 \mathrm{~mA}$.

\section{DyNAMiC ChaRACTERIZATION}

The dynamic characterization setup is shown in Fig. 6 The hybrid III-V/Si DFB laser was biased at $138 \mathrm{~mA}$ and directly modulated at $25 \mathrm{~Gb} / \mathrm{s}$ with a $2^{7}-1$ non-return-to-zero (NRZ) pseudo-random binary sequence (PRBS) generated by a bit pattern generator with a peak-to-peak voltage of $3.4 \mathrm{~V}$. After direct modulation, the optical signal was coupled to the silicon MRR for ER enhancement through optical filtering. The laser bias current was adjusted to match the MRR resonance for suppression of the low-frequency content of the modulated optical spectrum, as shown in Fig. 7 Thermal tuning of the MRR with heaters could lead to the same result [11]. After ER enhancement by the MRR, the optical signal was transmitted over up to $2.5 \mathrm{~km}$ of SSMF and received by a standard preamplified receiver connected to an error analyzer for bit-errorratio (BER) measurements and to a sampling oscilloscope for eye diagram monitoring.

The recorded eye diagrams are shown in Fig. 8 Considering first the back-to-back (B2B) scenario, it is possible to observe how the modulation ER is enhanced by the suppression of the signal ' 0 ' level by the MRR. The ' 0 ' level decreases getting closer to the yellow dashed line showing the oscilloscope ground level. This corresponds to an enhanced eye opening and an improvement in the ER from $3.8 \mathrm{~dB}$ to $6.8 \mathrm{~dB}$. Furthermore, considering eye diagrams after transmission, it is clear how the MRR filtering also enhances the signal dispersion tolerance. In fact, even if the dispersion effects are visible in all the eye diagrams, for the MRR filtered signal the eye remains open for a transmission distance up to $2.5 \mathrm{~km}$. Without MRR filtering, instead, the eye is almost closed already after transmission over $1 \mathrm{~km}$ of SSMF and becomes completely closed after $2 \mathrm{~km}$. 


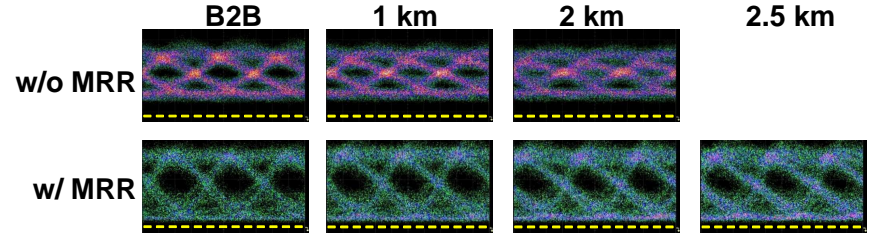

Fig. 8. Eye diagrams of the $25 \mathrm{~Gb} / \mathrm{s}$ signal for back-to-back and after transmission over 1, 2 and $2.5 \mathrm{~km}$ of SSMF with and without MRR filtering. The yellow dashed line highlights the oscilloscope ground level.

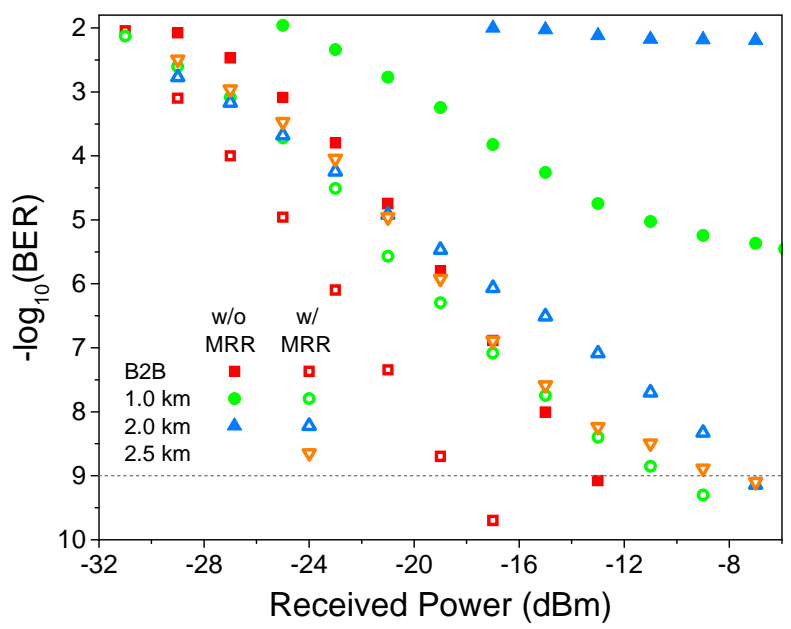

Fig. 9. Bit-error-ratio curves of the 25-Gb/s signal for back-to-back and after transmission over 1, 2 and $2.5 \mathrm{~km}$ of SSMF with and without MRR filtering.

These results are confirmed by the BER curves shown in Fig. 9 Considering first the $\mathrm{B} 2 \mathrm{~B}$ scenario, the receiver sensitivity, defined as the received power required for a $\mathrm{BER}=10^{-9}$, is improved by approximately $5 \mathrm{~dB}$ as a consequence of the ER enhancement provided by the MRR. After transmission over SSMF, the accumulated dispersion degrades the quality of the received signal both with and without MRR filtering. However, with MRR filtering, error free performance $\left(\mathrm{BER}<10^{-9}\right.$ ) can be achieved for a transmission reach up to $2.5 \mathrm{~km}$ of SSMF. Without MRR filtering, instead, the BER curves for transmission over 1-km and 2-km SSMF show that error-free performance cannot be achieved and error floors are measured above a $\mathrm{BER}=1 \times 10^{-6}$ and $\mathrm{BER}=5 \times 10^{-3}$, respectively. FEC could be employed at the receiver side, however, at the expense of increasing both latency and energy consumption.

In the case of 2.5-km SSMF transmission with the MRR, the measured power penalty at $\mathrm{BER}=10^{-9}$ is approximately $10 \mathrm{~dB}$ and $4 \mathrm{~dB}$ compared to the back-to-back BER values with and without MRR filtering, respectively. Even if such power penalty is significant and highlights the detrimental impact of dispersion, a receiver sensitivity as low as $-8 \mathrm{dBm}$ could still be achieved for a distance not reachable without the use of the MRR.

From Fig. 9 it is possible to notice that the performance for $2.5-\mathrm{km}$ SSMF is slightly better than for $2 \mathrm{~km}$. This behavior may be associated to instabilities in the measurement setup, including some drift in coupling the light out of the hybrid
DFB laser, which, through reflections, creates feedback into the laser cavity. This problem will clearly be solved by the integration of the DFB laser and MRR in a single device.

\section{CONCLUSION}

A transmitter for short reach application based on a hybrid III-V/SOI DFB DML operating at $25 \mathrm{~Gb} / \mathrm{s}$ was demonstrated by enhancing the DML modulation ER and dispersion tolerance through offset filtering using a silicon MRR. Error-free transmission $\left(B E R<10^{-9}\right)$ over $2.5-\mathrm{km}$ SSMF was achieved without the use of electronic equalization techniques, FEC or dispersion compensation. As both DFB laser and MRR have been fabricated on the SOI platform, combining the two devices can provide a compact all-silicon transmitter suitable for data center applications.

\section{ACKNOWLEDGMENT}

This research was funded by the European Commissions Seventh Framework Program under grant agreement no. 619626 SEQUOIA and supported by CoE SPOC, ref. DNRF123, DFF-4005-00558 project NANO-SPECs and by Vetenskapsrädet project PHASE, grant no. 2016-04510.

\section{REFERENCES}

[1] http://www.ieee802.org/3/cc/P802_3cc_Objectives.pdf

[2] http://www.ieee802.org/3/ca/public/meeting_archive/2016/03/harstead.pdf

[3] N. Nakahara, et al., "Direct modulation at 56 and $50 \mathrm{~Gb} / \mathrm{s}$ of $1.3 \mu \mathrm{m}$ InGaAlAs ridge-shaped-BH DFB laser," IEEE Photon. Technol. Lett., vol. 27, no. 5, pp. 534-536 (2015).

[4] S. M. R. Montaghiannezam, et al., "Single chip 52 Gb/s PAM4 transmission through -58 and $+10 \mathrm{ps} / \mathrm{nm}$ chromatic dispersion using directly modulated laser," in Proc. OFC, Th2A.59 (2016).

[5] D. Mahgerefteh, Y. Matsui, X. Zheng, and K. McCallion, "Chirp managed laser and applications," IEEE J. Sel. Topics Quantum Electron., vol. 16, no. 5, pp. 1126-1139 (2010).

[6] Z. Li, L. Yi, X. Wang, and W. Hu, " $28 \mathrm{~Gb} / \mathrm{s}$ duobinary signal transmission over $40 \mathrm{~km}$ based on $10 \mathrm{GHz}$ DML and PIN for $100 \mathrm{~Gb} / \mathrm{s}$ PON," Opt. Express, vol. 23, no. 16, pp. 20249-20256 (2015).

[7] Y. An, A. L. Riesgo, J. Seoane, Y. Ding, H. Ou, and C. Peucheret, "Transmission property of directly modulated signals enhanced by a micro-ring resonator," in Proc. OECC, 6F3-3 (2012).

[8] V. Cristofori, et al. "Error-free dispersion-uncompensated transmission at $20 \mathrm{~Gb} / \mathrm{s}$ over SSMF using a hybrid III-V/SOI DML with MRR filtering" in Proc. CLEO, STu1G.4 (2016).

[9] V. Cristofori, et al. "Direct modulation of a hybrid III-V/Si DFB laser with MRR filtering for $22.5-\mathrm{Gb} / \mathrm{s}$ error-free dispersion-uncompensated transmission over 2.5-km SSMF" in Proc. ECOC, Th.2.P2.SC4.42 (2016).

[10] A. Shen, et al., " $50 \mathrm{~km}$ error free transmission of fully integrated chirpmanaged $10 \mathrm{~Gb} / \mathrm{s}$ directly modulated C-band tunable III-V SOI hybrid lasers," in Proc. OFC, M2C.5 (2016).

[11] Y. Yokoyama, et al., "10.709-Gb/s-300-km Transmission of PLCbased Chirp-Managed Laser Packaged in Pluggable Transceiver Without Any Optical or Electrical Dispersion Compensation," in Proc. ECOC, We.1.C.4 (2008).

[12] G.-H. Duan, et al., "Hybrid III-V on silicon lasers for photonic integrated circuits on silicon," IEEE J. Sel. Topics Quantum Electron., vol. 20, no. 4, pp. 158-170 (2014).

[13] T. L. Koch and R. A. Linke, "Effect of nonlinear gain reduction on semiconductor laser wavelength chirping," Appl. Phys. Lett., vol. 48, no. 10, pp. 613-615 (1986).

[14] R. Stoffer, K. R. Hiremath, M. Hammer, L. Prkna, and J. Ctyroky, "Cylindrical integrated optical microresonators: Modeling by 3-D vectorial coupled mode theory," Opt. Commun., vol. 256, no. 1-3, pp. 46-67 (2005).

[15] Y. Ding, H. Ou, and C. Peucheret, "Ultra-high-efficiency apodized grating coupler using fully etched photonic crystals," Opt. Lett., vol. 38, no. 15 , pp. 2732-2734 (2013). 